\section{FORTRAN IV functions to compute expected normal scores}

\section{WILLIAM P. DUNLAP and SUSAN G. BROWN Tulane University, New Orleans, Louisiana}

Fisher and Yates (1963) were the first to propose the use of expected normal scores to provide numerical values in cases in which only ordinal information was known, thus permitting the use of techniques such as analysis of variance. They also suggested the use of expected normal scores to replace data that did not conform to assumptions of tests of significance, thus presaging what are known today as the "normal scores tests." The normal scores tests are a class of nonparametric techniques that have the remarkable property of asymptotic relative efficiency of greater than or equal to 1 when compared with Student's t test (Chernoff \& Savage, 1958; Gastwirth \& Wolff, 1968). This means that when sample sizes are large, the normal scores tests have the power to detect differences between means that are equal to (when assumptions are met) or greater than (when assumptions are not met) their parametric counterparts. Therefore, as nonparametric tests, the normal scores tests are, in most cases, better than nonparametric tests that replace the raw data with ranks, and their power compares quite favorably with that of corresponding parametric tests. Why, then, are these tests so seldom used? Two probable answers are that ranks are simpler to work with and that adequate tables of expected normal scores are not generally available. Tables for samples up to 25 were published by Fisher and Yates (1963), up to 50 by Owen (1962) and Pearson and Hartley (1954), up to 100 by Harter (1961), and up to 200 by Harter (1970). Because of their bulk, extensive tabulations do not appear even in statistics textbooks that deal primarily with nonparametric statistics.

The need for expected normal scores for large sample sizes becomes particularly apparent when special applications such as those outlined by Stavig and Acock (1976) are considered. Stavig and Acock described a procedure for assessing the significance of correlation coefficients in a matrix. The number of expected normal scores required is two times the number of unique correlations in the matrix. Thus, with 50 intercorrelated variables, normal scores based on a total sample of 2,450 are required. Although Stavig and Acock suggest the use of an approximation procedure, Harter (1961) showed that, as it stands, this approximation is inadequate.

The present paper describes two FORTRAN function subprograms that compute expected normal scores.

The authors' mailing address is: Department of Psychology, Tulane University, New Orleans, Louisiana 70118.
The first function, SCR, provides a very quick approximate solution, accurate to three decimal places (see Appendix A). This first function should be sufficient for most applications (e.g., normal scores tests). The second function, SCOR, computes expected normal scores directly, using numerical methods (see Appendix B). Although the latter solution is costly in terms of computer time, the results are accurate to at least five decimal places, and the computations agree in all places with Harter's (1970) tabulations.

Function SCR. This short approximate solution for expected normal scores is based on an approximating algorithm originally proposed by Blom (1958). Harter (1961) described corrections for the Blom algorithm that improve its accuracy to no worse than one digit error in the third decimal place. Because the Blom algorithm requires inverses of the normal probability integral, function SCR obtains these inverses from a quick approximate function that is also accurate to three decimal places and that was described earlier in this journal by Emerson (1979). Although Harter (1961) felt that using the improved Blom algorithm for $\mathrm{N}$ greater than 400 should be emphatically discouraged, we have found that, by comparing the output of SCR with the more accurate direct solution to be described next, SCR maintains its three-decimal-point accuracy up through $\mathrm{N}=3,000$. Therefore, $\mathrm{SCR}$ should be of sufficient accuracy and range for most practical applications.

Function SCOR. The second function, SCOR, solves for the expected value of the Jth of $\mathrm{N}$ normal scores by direct numerical integration of the equation

$$
E\left(x_{J / N}\right)=\frac{N !}{(N-J) !(J-1) !} \times P^{(N-J)} Q^{(J-1)} Z d x,
$$

where $\mathrm{P}$ is the proportion below $\mathrm{x}$ and $\mathrm{Q}$ the proportion above $\mathrm{x}$ on the standard normal curve and $\mathrm{z}$ is the ordinate of the standard normal curve at $x$. The quantities $P$ and $Q$ are computed by a double precision function, POFZ, which solves the integral of the normal distribution from minus infinity to $\mathrm{x}$, based on Algorithm 26.2.11 from Zelen and Severo (1965, p. 932). The outer integral in the above equation is solved as follows. First, function SCR is used to approximate the center of the distribution of the Jth of $\mathrm{N}$ normal scores. Next, Simpson's rule is used to integrate the distribution above this central value, such that the integration is stopped when the addition of further points no longer increases the cumulative area stored in single precision. Then the area below the estimated central value is computed by a similar Simpson integration. The sum of these two integrals is the expected normal score. Expected normal scores computed by SCOR agree completely with Harter's (1970) five-place tables. 
Time Requirements. Function SCR computes a single expected normal score in approximately $0.25 \mathrm{msec}$ on the DEC.20 computer. The time of computation is essentially independent of the sample size, $\mathrm{N}$, and the ordinal position, J. Computation times for SCOR, on the other hand, are much longer and depend upon both $\mathrm{N}$ and $\mathrm{J}$. The longest times for SCOR occur when $\mathrm{J}=1$ or $\mathrm{J}=\mathrm{N}$, and are in the neighborhood of $400 \mathrm{msec}$. The shortest times occur when $J=N / 2$, and range from about $100 \mathrm{msec}$ for large $\mathrm{N}$ to about $200 \mathrm{msec}$ for small $\mathrm{N}$ on the DEC-20.

Program Availability. Functions SCR and SCOR are written in standard FORTRAN IV and run on a DEC-20 computer. Although SCR is written entirely in single precision, SCOR uses double precision functions and storage. The use of double precision in SCOR was only necessary for very large values of $\mathrm{N}$, so that if only single precision were used, SCOR would have fiveplace accuracy for almost any practical problem. Because the language used is standard, these functions should run without change on any computer with a FORTRAN IV compiler. An eight-statement main program is included at the front of each function for instructive purposes. These main programs contain some DEC-specific FORTRAN statements; however, users of these functions will probably wish to write their own main routines, depending upon the particular use they have in mind.

Neither function uses dimensioned storage; therefore, memory size need only be sufficient to load the functions together with main routines to run them. Listings of the functions may be obtained at no cost from William P. Dunlap, Department of Psychology, Tulane University, New Orleans, Louisiana 70118.

\section{REFERENCES}

Bцом, G. Statistical estimates and transformed beta-variables. New York: Wiley, 1958.

Chernoff, H., \& Savage, I. R. Asymptotic normality and efficiency of certain nonparametric test statistics. Annals of Mathematical Statistics, 1958, 29, 972-994.

Emerson, P. L. Computer approximation of the inverse of the normal distribution function. Behavior Research Methods \& Instrumentation, 1979, 11, 397-398.

Fisher, R. A., \& YATES, F. Statistical tables for biological, agricultural, and medical research. New York: Hafner, 1963.

GastwirTh, J. L., \& WolfF, S. An elementary method for obtaining lower bounds on the asymptotic power of rank tests. Annals of Mathematical Statistics, 1968, 39, 2128-2130.

Harter, H. L. Expected values of normal order statistics. Biometrika, 1961, 48, 151-165.

Harter, H. L. Order statistics and their use in testing and estimation (Vol. 2). Washington, D.C: U.S. Government Printing Office, 1970.

Owen, D. B. Handbook of statistical tables. Reading, Mass: Addison-Wesley, 1962.

Pearson, E. S., \& Hartley, H. O. Biometrika tables for statisticians. London: Cambridge University Press, 1954.

Stavig, G. R., \& Acock, A. C. Evaluating the degree of dependence for a set of correlations. Psychological Bulletin, 1976, 83, 236-241.

Zelen, M., \& Severo, N. C. Probability functions. In M. Abramowitz \& I. A. Stegun (Eds.), Handbook of mathematical functions. New York: Dover, 1965.

Appendix A

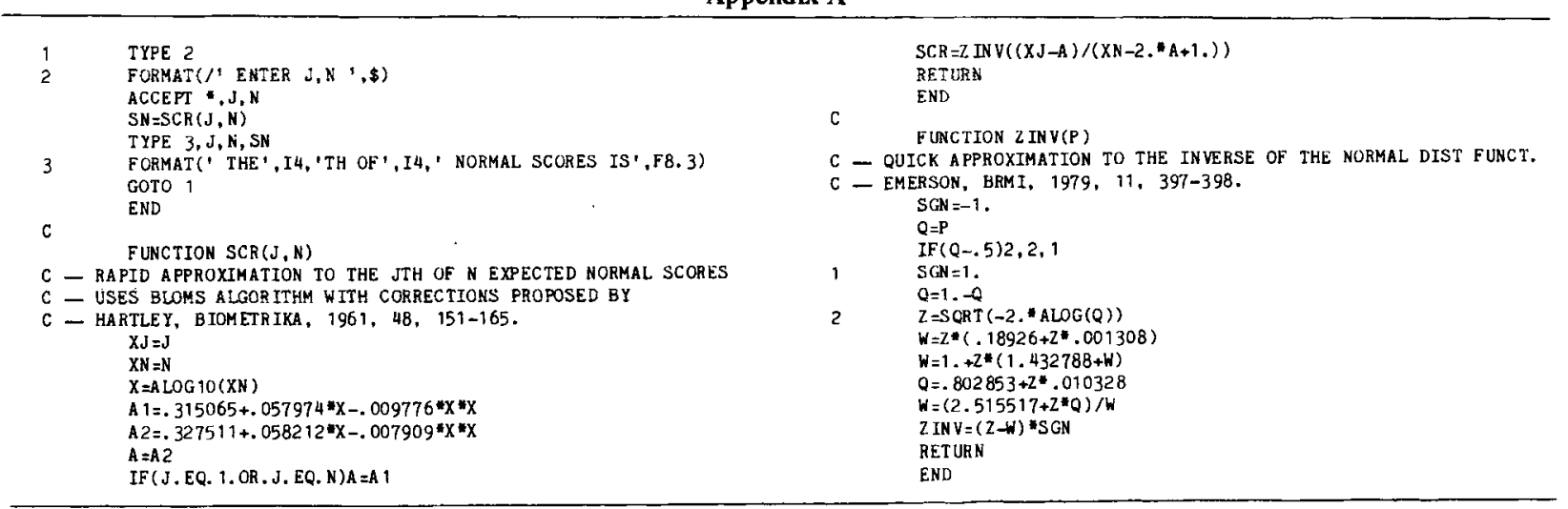

Appendix B

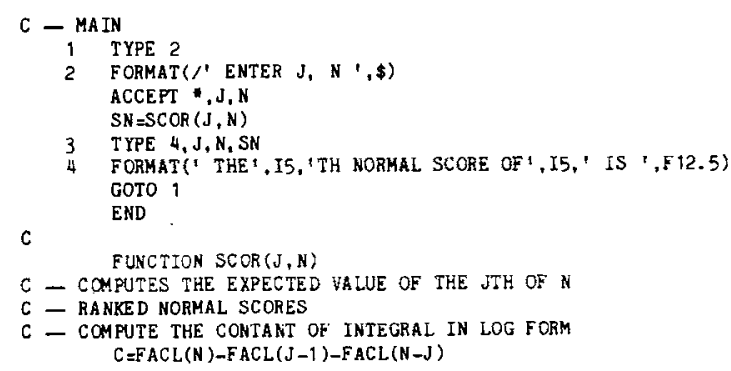

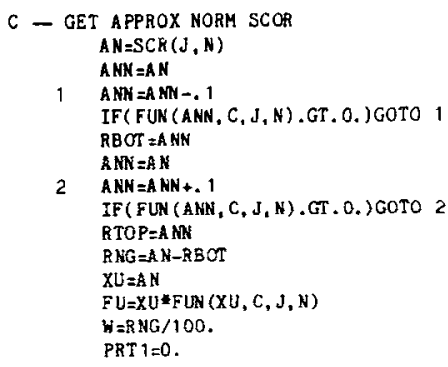



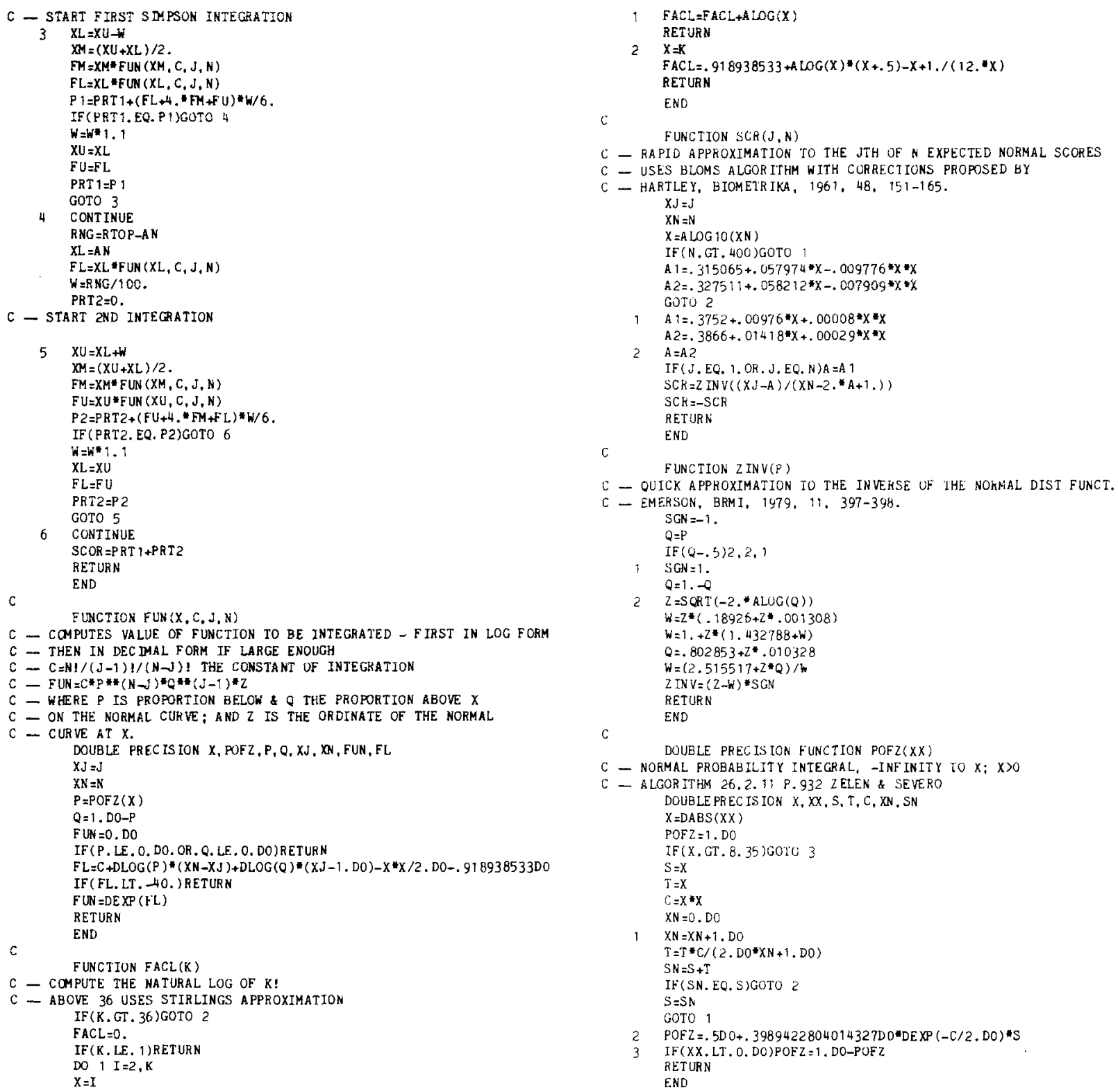

(Manuscript accepted for publication May 6, 1983.)

\section{ERRATUM}

Przeorek, J., Sanchez, R., \& Deni, R. BASIC-PLUS-2 programs to perform experimental procedures for paired associate verbal learning (Behavior Research Methods \& Instrumentation, 1983, 15, 93-94)-The surname of the first author of this article was misspelled. The name should correctly read Janus Przeorek. 